\title{
A Successful implementation of an idea to a nationally approved plan: Analyzing Iran's National Health Roadmap using the Kingdon model of policymaking
}

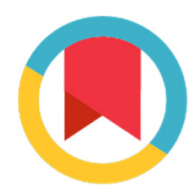

\begin{abstract}
Hassan Hashemi ${ }^{1}$, Ali Akbar Haghdoost ${ }^{2}$, Mohammad Haji-Aghajani ${ }^{3}$, Ghasem Janbabaee ${ }^{4}$, Ali Maher ${ }^{5}$, Somayeh Noori Hekmat ${ }^{6}$, Amir M. Javadi ${ }^{7}$, Rohaneh Rahimisadegh ${ }^{8}$, Samira Emadi ${ }^{9}$, Mahamad Reza Rajabalipour ${ }^{10}$, Hajar Haghighi ${ }^{11}$, Reza Dehnavieh ${ }^{12}$, Masoud Ferdosi ${ }^{13}$, Gholamreza Khademi ${ }^{14}$, Mohammad Hossein Mehralhasani ${ }^{15}$, Asma Sabermahani ${ }^{16}$, Kaveh Nouhi Bezanjani ${ }^{17}$, Abedin Iranpour ${ }^{18}$, Hamidreza RashidiNejad ${ }^{19}$, Fatemeh Moeen Samadani ${ }^{20}$, Maryam Maki ${ }^{21}$, Behzad Kalantari ${ }^{22}$, Nahid farrokhyar ${ }^{23}$, Hamed Rouhanizadeh ${ }^{24}$, Monireh Falakbaz ${ }^{25}$, Hamid Allahyari ${ }^{26}$, Mohammad Taghi Fathalian ${ }^{27}$, Ali Reza Khajehmirzaei ${ }^{28}$, Ali Jannati ${ }^{29}$, Javad Derakhshani ${ }^{30}$, Ali Rezapour ${ }^{31}$, Jamal Eftekhari ${ }^{32}$, Parisa Khaterneshanian fam ${ }^{33}$, Mahmoud Kazemi ${ }^{34}$, Mohsen Mohammadi ${ }^{35}$, Payam Rastbin ${ }^{36}$, Bahareh Pirhayati ${ }^{37}$, Elahe Souri ${ }^{38}$, Amin Torabipour ${ }^{39}$, Mohammad Keshvari ${ }^{40}$, Najmeh Alikhani ${ }^{41}$, Lobat Salehi' ${ }^{42}$, Mohammad Moradi ${ }^{43}$, Saeed Aghajaniyan ${ }^{44}$,Arvin Hedayati ${ }^{45}$, Maryam Kargar ${ }^{46}$, Leila Sharifi ${ }^{47}$, Habib OmraniKhoo ${ }^{48}$, Abdollah Poursamad ${ }^{49}$, Noraste Amrolahi boyuki ${ }^{50}$, Zahra Zarei ${ }^{51}$, Zohre Sahraei ${ }^{52}$,Abdollah Keshavaez ${ }^{53}$, Mahdiyeh Sadeghi ${ }^{54}$, Somayae Abdollahi Sabet ${ }^{55}$,Katayon Taiari ${ }^{56}$, Jalil Koohpayehzadeh ${ }^{57}$, Elham Moazam ${ }^{58}$, Abbasali Dehghani Tafti ${ }^{59}$, Seyyedeh Somaye Forghani Dehnavi ${ }^{60}$, Mostafa Rezaei Barownaghi ${ }^{61}$, Hossein Jafari62, Somayeh Foroghifar ${ }^{63}$, Masoud Arefnejad ${ }^{64}$, Hossein Ebrahimipour ${ }^{65}$, Mojtaba Sedaghat ${ }^{66}$, Saeedeh Mehdipoor ${ }^{67}$, Fariba Mollajafari ${ }^{68}$, Reza Forouzan ${ }^{69}$, Saeed Meskini Mood ${ }^{70}$, Shrvin Sharifpour ${ }^{71}$, Amin Adel ${ }^{72}$, Shabnam Nirouand ${ }^{73}$, Gholam Reza Ghaznavi ${ }^{74}$, Reza Zamanzadeh ${ }^{75}$, Khatereh Etesam ${ }^{76}$, Mahdieh Dalili"77, Mohamad Jafari Sirizi ${ }^{78}$, Roodabeh Omidifar ${ }^{79}$
\end{abstract}

Received: 12 Jan 2018

Published: 6 June 2018

\section{Abstract}

Introduction: Hospital beds, human resources, and medical equipment are the costliest elements in the health system and play an essential role at the time of treatment. In this paper, different phases of the NEDA 2026 project and its methodological approach were presented and its formulation process was analysed using the Kingdon model of policymaking.

Methods: Iran Health Roadmap (NEDA 2026) project started in March 2016 and ended in March 2017. The main components of this project were hospital beds, clinical human resources, specialist personnel, capital medical equipment, laboratory facilities, emergency services, and service delivery model. Kingdon model of policymaking was used to evaluate NEDA 2026 development and implementation. In this study, all activities to accomplish each step in the Kingdon model was described.

Results: The followings were done to accomplish the goals of each step: collecting experts' viewpoint (problem identification and definition), systematic review of the literature, analysis of previous experiences, stakeholder analysis, economic analysis, and feasibility study (solution appropriateness analysis), three-round Delphi survey (policy survey and scrutinization), and intersectoral and interasectoral agreement (policy legislation).

Conclusion: In the provision of an efficient health service, various components affect each other and the desired outcome, so they need to be considered as parts of an integrated system in developing a roadmap for the health system. Thus, this study demonstrated the cooperation process at different levels of Iran's health system to formulate a roadmap to provide the necessary resources for the health sector for the next 10 years and to ensure its feasibility using the Kingdon policy framework.

Keywords: Policy making, National Roadmap, Health system, Iran

Copyright $₫$ Iran University of Medical Sciences

Cite this article as: Hashemi H, Haghdoost AA, Noori Hekmat S, Haji-Aghajani M, Janbabaee G, Maher A, et al. A Successful implementation of an idea to a nationally approved plan: Analyzing Iran's National Health Roadmap using the Kingdon model of policymaking. Med J Islam Repub Iran 2018(6 June);32:46. https://doi.org/10.14196/mjiri.32.46

\section{Introduction}

Health system resources are costly and require considerable capital investment. Developing the resources takes time and requires substantial investment and forecasting. From this standpoint, the most important components of the health sector include hospital facilities, human resources, and major medical equipment (1). Forecasts indicate that new innovations in the field of health technologies, shifts in the burden of diseases, and demographic changes are the most important trends affecting the society's expectations of the health system, and thus increasing its costs (2). An increase in the incidence and prevalence of multi-organ diseases leads to an increased need for more sophisticated diagnostic provisions and treatment services that are highly expensive. Furthermore, it is predicted that with an ageing population (increased lifeexpectancy), the need for adequate health services will increase even further.

The provision of hospital beds and the necessary facilities, the required human resources, and medical equipment form the most capital intensive parts of a health system (3). These resources are the most important components of the health service chain and play an important role at the point of service delivery (treatment). Considering the importance of the interdependency of these components, 
they should be considered as parts of an integrated system when developing a roadmap for the health system.

The provision and the availability of hospital beds are the primary units of service delivery capacity in a region and determine the required level of other necessary resources in the health sector (4). Thus, an equal distribution of hospital beds is one of the most important requirements in the health sector. Having the foresight to provide a fair

1- Faculty of Medicine, Tehran University of Medical Sciences, Tehran, Iran. 2- Modeling in Health Research Center, Institute for Future Studies in Health, Kerman University of Medical Sciences, Kerman, Iran. 3- Faculty of Medicine, Shahid Beheshti University of Medical Sciences, Tehran, Iran. 4- Department of Hematology-Oncology, Gastrointestinal Cancer Research Center, Faculty of Medicine, Mazandaran University of Medical Sciences, Sari, Iran. 5- Department of Health Policy, School of Medical Education, Shahid Beheshti University of Medical Sciences, Tehran, Iran. 6- Modeling in Health Research Center, Institute for Futures Studies in Health, Kerman University of Medical Sciences, Kerman, Iran. 7- Executive director, Iranian healthcare information Observatory, Researcher of Noor Ophthalmology Research Center, Tehran, Iran. 8- Research Center for Health Services Management, Institute for Futures Studies in Health, Kerman University of Medical Sciences, Kerman, Iran. 9- Research Center for Health Services Management, Institute for Future Studies in Health, Kerman University of Medical Sciences, Kerman, Iran. 10- Supervisor of Public Health Department in School of Public Health in Bam University of Medical Sciences, Bam, Iran. 11- Research Center for Health Services Management, Future Studies Research Center in Health, Kerman University of Medical Sciences, Kerman, Iran. 12- Medical Informatics Research Center, Institute for Futures Studies in Health, Kerman University of Medical Sciences, Kerman, Iran. 13- Health Management and Economics Research Center (HMERC), Isfahan University of Medical Sciences, Isfahan, Iran. 14Assistant Director of Medical Accident and Emergency Management Center, Kerman University of Medical Sciences, Kerman, Iran. 15- Medical Informatics Research Center, Institute for Futures Studies in Health, Kerman University of Medical Sciences, Kerman, Iran. 16- Research Center for Health Services Management, Institute for Future Studies in Health, Kerman University of Medical Sciences, Kerman, Iran. 17- Master of Public Administration, Research Center for Health Services Management, Institute for Future Studies in Health, Kerman University of Medical Sciences, Kerman, Iran. 18- HIV/STI Surveillance Research Center, and WHO Collaborating Center for HIV Surveillance, Institute for Futures Studies in Health, Kerman University of Medical Sciences, Kerman, Iran. 19- Research Center for Modeling in Health, Institute for Future Studies in Health, Kerman University of Medical Sciences, Kerman, Iran. 20-Department of Statistics, Deputy of Curative Affaire, Kerman University of Medical Sciences, Kerman, Iran. 21-Department of Environmental Health, School of public Health, Kerman University of Medical Sciences, Kerman, Iran. 22MD, Department of Planning, Deputy of Curative Affaire, MOHME, Tehran, Iran. 23- Department of Planning, Deputy of Curative Affaire, MOHME, Tehran, Iran. 24- Department of Pediatrics, Mazandaran University of Medical Sciences, Sari, Iran. 25Department of Statistics, Deputy of Curative Affaire, Guilan University of Medical Sciences, Rasht, Iran. 26- Golestan University of Medical Sciences, Gorgan, Iran. 27-Management of Diseases, Diagnosis \& Treatment Center, Semnan University of Medical Sciences, Semnan, Iran. 28-MD, vice-chancellery of treatment, Shahroud University of Medical Sciences, Shahroud, Iran. 29Iranian Center of Excellence in Health Management (IceHM), School of Management and Medical Informatics, Tabriz University of Medical Sciences, Tabriz, Iran. 30- Health Information Officer, Tabriz University of Medical Sciences, Tabriz, Iran. 31- Human Resources. Head of Hospital Administration and Clinical Services Excellence. Ardabil University of Medical Sciences, Ardabil, Iran. 32- Department of clinical affairs, Urmia university of medical sciences, Urmia, Iran. 33- Head of hospital administration and clinical services excellence. Treatment dept. Faculty of medical sciences and health services, Maragheh. Maragheh, Iran. 34Department of Statistics, Deputy of Curative Affaire, Kermanshah University of Medical Sciences, Kermanshah, Iran. 35- Student Research Committee, Kermanshah University of Medical Sciences, Kermanshah, Iran. 36- Department of Statistics, Deputy of Curative Affaire, medical university of Kurdestan, Iran. 37- Expert Statistics, Unit of Statistics, Ilam University of Medical Science, Ilam, Iran. 38- Deputy of Curative Affaire, Hamadan University of Medical Science, Hamadan, Iran. 39- Department of Health Services Management, School of Health, Ahvaz Jundishapur University of Medical Sciences, Ahvaz, Iran. 40-Department of Health Services Management, School of Medical Management and Information Sciences, Iran University of Medical Sciences, Tehran, Iran. 41- Faculty of Management and Economics, Science and Research Branch, Islamic Azad University, Tehran, Iran. 42Head of The Policy and Strategic Planning Division, the Chancellor and Chief Executive Office, Shiraz University of Medical Sciences. 43- Senior expert in the policy-making and planning center of Shiraz University of Medical Sciences, Shiraz University of Medical Sciences, Shiraz, Iran. 44- Shiraz University of Medical Sciences. 45- Shiraz University of Medical Sciences, Shiraz, Iran. 46- Jahrom University of Medical Sciences, Jahrom, Iran. 47- Nursing Director of Bandar Abbas University of Medical Sciences, Iran. 48-Department of Public Health, Faculty of health, Bushehr University of Medical Sciences, Bushehr, Iran. 49- Yasuj University of Medical Science, Yasuj, Iran. 50- Head of Administrative Affairs and Human Resources Department of Treatment, Alborz University of Medical Science, Alborz, Iran. 51- Qum University of Medical Sciences. Qum. Iran. 52-Vice-Chancellor in Treatment Affairs, Qazvin University of Medical Science, Qazvin, Iran. 53-Vice-Chancellor in Treatment Affairs, Qazvin University of Medical Science, Qazvin, Iran. 54- Arak university of medical science, Arak, Iran. 55- Department of Community Medicine, Faculty of Medicine Zanjan University of Medical Sciences, Zanjan, Iran. 56- Deputy of Curative Affaire, Saveh university of medical sciences, Saveh. Iran. 57- Preventive Medicine \& Public Health Research Center, Iran University of Medical Sciences, Tehran, Iran. 58Cancer Prevention Research Center, Isfahan University of Medical Science, Iran. 59- School of Public Health, Shahid Sadoughi University of Medical Sciences, Yazd, Iran. 60-Shahrekord University of Medical Sciences, Shahrekord, Iran. 61- Kashan University Medical Science, Iran. 62-Department of Statistics, Deputy of Curative Affaire, Rafsanjan University of Medical Sciences, Rafsanjan, Iran. 63-Department of Statistics, Deputy of Curative Affaire, Bam University of Medical Sciences, Kerman, Iran. 64- Department of Statistics, Deputy of Curative Affaire, Zabol University of Medical Sciences, Zabol, Iran. 65- Social Determinants of Health Research Center, Mashhad University of Medical Sciences, Mashhad, Iran. 66- Department of Community Medicine, Faculty of Medicine, Tehran University of Medical Sciences, Tehran, Iran. 67- Colorectal Research Center, Iran University of Medical Sciences, Tehran, Iran. 68-Vice-Chancellor's Office in Treatment Affairs, Health Economy, Standard and Health Technology Office, Shahid Beheshti University of Medical Sciences, Tehran, Iran. 69- Deputy of Curative Affaire, Sabzevar University of Medical Sciences, Sabzevar, Iran. 70- Deputy of Curative Affaire, Zahedan University of Medical Sciences, Zahedan, Iran. 71Deputy treatment, Babol University of Medical Sciences, Babol Iran. 72- Department of management sciences and health economics, school of Health, Mashhad University of Medical Sciences, Mashhad, Iran. 73- Faculty of Medicine, Mashhad University of Medical Sciences, Mashhad, Iran. 74- Department of Statistics, Deputy of Curative Affaire, Jiroft University of Medical Sciences, Jiroft, Iran. 75- North Khorasan University of Medical Sciences, Bojnurd, Iran. 76- The Coordination Office for Insurance, Tariffs and Standards, Birjand University of Medical Sciences, Birjand, Iran. 77- The Coordination Office for Insurance, Tariffs and Standards, Kerman University of Medical Sciences, Kerman, Iran. 78- Kerman Insurance Organization, Kerman, Iran. 79- Lorestan University of Medical Sciences, Khorramabad, Iran.

$\uparrow$ What is “already known” in this topic:

This study demonstrated the cooperation process at different levels of Iran's health system to formulate a roadmap to develop the necessary resources for the health sector for the next 10 years and to ensure its feasibility using the Kingdon policy framework.

$\rightarrow$ What this article adds: Prior to this project, no study was conducted to estimate the structural resources of the health sector in Iran. 


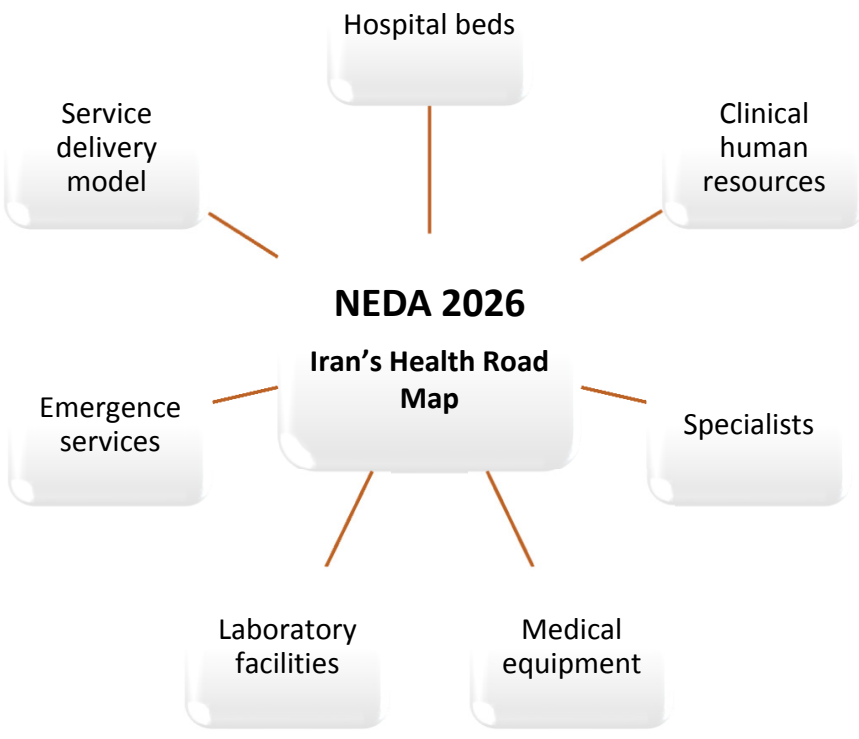

Fig .1 .Iran's National Health Roadmap Components

and economically viable expansion of hospital beds is the first step in the decision-making process to expand other expensive resources of the health system (5).

Human resources is one of the most important inputs of the health system, with a major impact on public health outcome, accounting for the largest share of funding in the health sector. Training competent and skilful manpower is time-consuming and costly (6). However, maintaining a good balance between the requirements of the supply and demand is crucial to minimize or prevent the wastage of resources. The trained and competent manpower should also be ready to meet the needs of the health system in the coming years. Accurate forecasting of the number of human resources needed in each speciality, along with competent conduct, will ensure efficient performance of the health system in the years ahead (7).

Furthermore, making decisions about the purchase and expansion of medical equipment (capital expenditure) is one of the major strategic decisions that should be considered in the health sector $(8,9)$. In most cases, these medical equipment and machinery are costly and expected to be in active use for at least 10 years to justify the expenditure (10). On the other hand, the pace of innovations in science and technology in general and in medical equipment in particular is so fast that the purchasing decision may coincide with the availability of newer versions of medical equipment with increased functionality at a lower price. Therefore, sufficient attention and consideration should be paid to the selection of health-related technologies to ensure that new equipment being considered are compatible with the existing equipment and can satisfy the future needs of the health system (11).

The distribution of health resources and the efficient and economic exploitation of these resources can cause contradictory issues in the health system. The available re- sources of a country, in addition to its GDP, are limited and only a small portion is allocated to the health sector. Therefore, all forecasting studies should consider the country's GDP growth fluctuations and its share of the health sector as one of the limitations when designing and implementing future scenarios.

\section{Iran's National Health Roadmap (NEDA 2026)}

\section{Components}

A balanced expansion of the available health resources is a prerequisite for reforms in the health services delivery system. Iran's Health Evolution Plan revealed that quality improvement was out of reach due to lack of necessary infrastructure, lack of sustainable resources, and lack of adequate availability of health care professionals, particularly in remote areas (see Fig. 1).

The Health Roadmap project was conducted for Kerman province by Kerman University of Medical Sciences (KMU) for the first time. After presenting Kerman Roadmap report, KMU was delegated to develop Iran's National Health Roadmap. The NEDA 2026 project commenced in March 2016 and ended in March 2017.

One of the targets of the NEDA 2026 project was to create a fair access to health care services in Iran. For the first time in Iran, the NEDA 2026 project was done to design and devise a master plan which would combine quantitative modelling with the experiences and predictions of experts in the health care field. The experts were asked to bear in mind future scenarios and estimate the resources needed for the provision of a health care service for the next 10 years.

In this project, the number of emergency beds as well as inpatient beds, the required level of medical manpower, nursing personnel, midwifery manpower, dentistry personnel, pharmacists, capital medical equipment needs, 

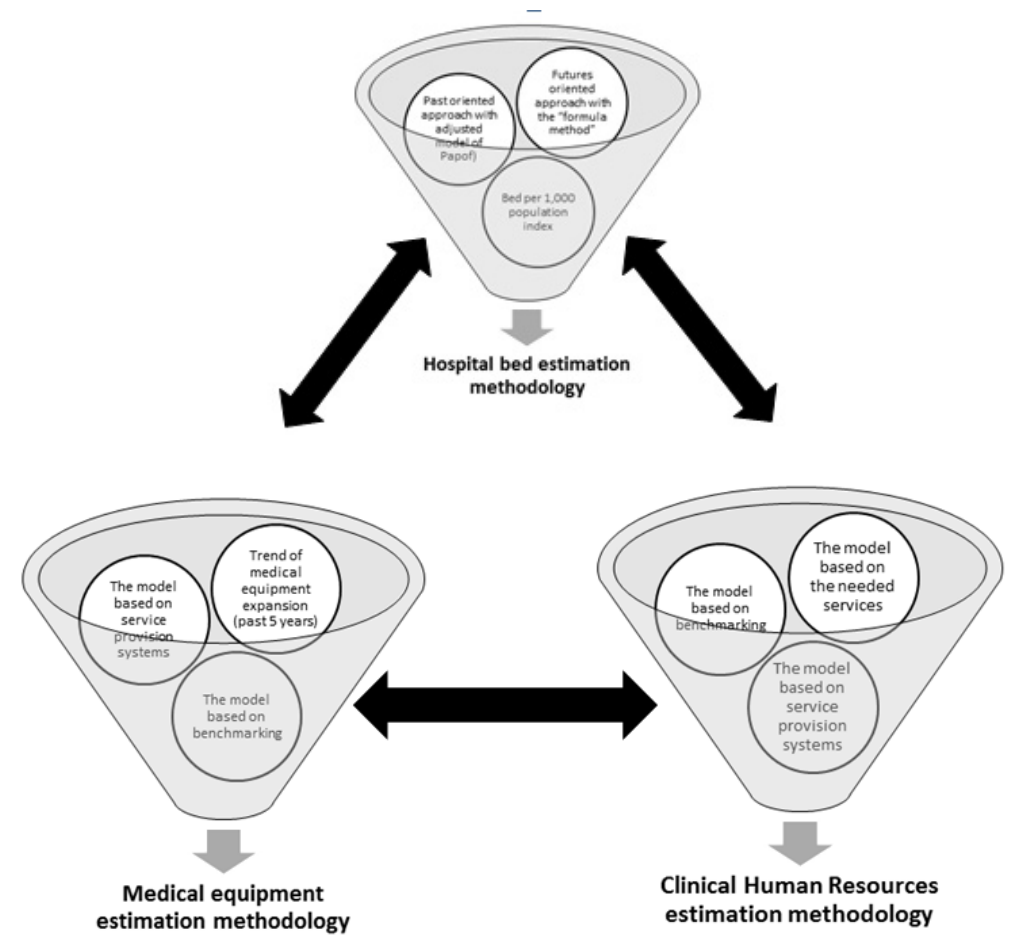

Fig. 2. Methodological approach of Iran's Health Roadmap

disasters, and emergency needs of 439 towns and cities across Iran were estimated. Using an economic simulation, the possible costs of implementing the roadmap were also estimated.

\section{Methodology}

\section{The phases of the NEDA project}

1- Capacity building: NEDA secretariats were organised in all medical universities nationwide, and the provincial collaboration offices were established in regional medical universities. To increase collaboration in different regions, regional offices were organized where 4 to 6 universities in a geographical area collaborated with one another to produce a joint roadmap. Regional medical universities hold workshops to familiarize the universities with the concepts involved.

2- Data collection: Data were collected on the present status of hospital beds, human resources, medical equipment, and the distribution and trend of hospital performance indicators. Moreover, other countries' experiences in developing master plans were reviewed, their resource allocation standards were studied, and benchmarking studies were performed.

3-Modelling: In this phase, the ranges of the model parameter variation were determined, and 3 national modelling workshops covering bed availability and allocation, human resources, and medical equipment were held. Moreover, resource forecasting panels at all medical universities were consulted. Triangulation of 3 cross check approaches was used to estimate the number of hospital beds and the clinical human resources needed (Figure 2).

4-Results validation: Because the modelling phase was performed separately by each university's secretary, a variation in modelling results between universities was expected. Therefore, fairness and equity indicators were chosen to evaluate the results. As equity is the most important indicator in the WHO model of health system functions and objectives, both horizontal and vertical equity were tested for all the resources that needed estimation. In vertical equity, the goal was to achieve the same resource per capita in the same city for needs; in horizontal equity, the goal was to assign the resource per capita in the same city for its status in the referral system.

\section{Results}

\section{NEDA 2026 outcomes}

The main objective of the project was to reduce the unnecessary migration to larger cities to receive basic health care. Estimates of the project will speed up the development of resources in small remote areas while controlling the resource allocation trend in large cities. Consequently, the implementation of the outcomes of this project will lead to a $30 \%$ increase in horizontal equity, a $23 \%$ decrease in the variance between provinces, and a reduction in the variance between the cities within each province (vertical equity).

\section{Analysing Iran's Health Roadmap formulation} process using the Kingdon model of policymaking

Developing the Health Roadmap of Iran was initiated as a small experiment in a province and is now considered as the nation's 10-year roadmap. This roadmap is the most important document that the Ministry of Health has adopted as its 10-year plan for health care in Iran. Different 
departments of Iran's Ministry of Health, including the deputy for education, the deputy for health affairs, and the deputy for resources supply, have agreed to cooperate in its implementation. This roadmap was also approved by the decision-making institutions at the national level. Many factors have contributed to the success of this health roadmap, which can be analysed using the Kingdon model of policymaking.

\section{Problem definition and conceptualization}

According to John Kingdon's theory of streams in the politics of policymaking, problem identification and definition is the first stage of policymaking (12). At the start of the study, the issue was carefully clarified and formulated by considering experts' viewpoint. The main issue was that some of the stakeholders did not fully take responsibility for rationing programs implementation and, in some cases, it was not the basis for health resource allocation decisions.

Furthermore, given the interdependency of the resources in the health care system, it is essential that they all be considered in the regionalisation of strategic resources. Strategic health care resources are those that are capital intensive (expensive) and whose deployment takes a considerable amount of time and planning.

On the other hand, for the continuity of "the services chain", especially in the field of health care services, all strategic resources should be considered for the provision of one particular service. This way, a quality and safe service is provided by efficient utilization of the resources. Manpower is the most important factor in the health care sector. The provision of service beds will be efficiently done only when the manpower is fit for the purpose, trained, and available. Providing safe and efficient manpower depends on support services and facilities the provision of medical equipment tailored for the needs of the patients. However, in the previous rationing programs of health care services in Iran, the main emphasis was on hospital beds, and other strategic resources and considerations were not taken into account.

\section{Appropriate solution}

Health care resources are highly valuable and expensive and their expansion and allocation require strong scientific evidence (13). For solution appropriateness analysis, a systematic review of the literature, analysis of previous experiences, stakeholder analysis, economic analysis, and a feasibility study were conducted.

To develop the modelling methodology of the required resources needed in the next 10 years, 3 systematic reviews were conducted, which were as follow: a systematic review of methodologies to estimate the number and distribution of hospital beds; a systematic review of methodologies to estimate the number and distribution of the health care personnel; and a systematic review of methodologies for health technology horizontal scanning. The evidence from these studies were used to design models to estimate the resources and determine the parameters.

In this study, previous experiences were also analysed.
Rationing has a partially long history in Iran. In the present study, documents of previous rationing programs were reviewed and the provincial and national experts' feedbacks were collected. In this analysis, the strengths and limitations of the previous rankings of rationing programs were investigated. Moreover, the reasons for the imprecise implementation of previous rationing programs in Iran were analysed.

In the economic analysis of Iran's Health Roadmap, the annual costs of the implementation of this project were estimated. In this analysis, the changing trend of the main economic parameters, such as fluctuations in the inflation rate, fluctuations in the annual wages and salaries, changes in the GDP, and the health care share of the GDP, were estimated. This analysis revealed that by assuming a $3 \%$ to $5 \%$ growth in GDP, allocating $7 \%$ to $9 \%$ of the GDP to fund health care costs, by the inflation rate of $10 \%$ to $14 \%$, an annual salary increase of $10 \%$ to $14 \%$, and by allocating $80 \%$ of the share of the health care budget to health services (both inpatient and outpatient treatment), this health roadmap can be implemented by 2026 in all cities across the country. The results of the economic analysis convinced many managers and governmental authorities outside the Ministry of Health to accept the Health Roadmap and the financial obligations incurred by its implementation.

\section{Policy survey and scrutinization}

The policy was scrutinized using experts' opinions. Also, in this study, experts at both the provincial and the national levels were involved to ensure the accuracy and fairness of this policy. Expert panels were selected in all provinces to predict the most probable and the best future scenarios. Further, a three-round Delphi survey was performed with a large group of experts to reach a consensus on the model of estimation and its parameters. Additionally, the experts took part in the validation and finalisation of the modelling findings relevant to their own provinces.

Customized policy recommendations on the NEDA outcomes were designed and forwarded to policymakers and stakeholders. A policy recommendation on health human resources supply and demand was designed for the deputy for education at the Ministry of Health and forwarded to the deputy for education. Methodologies and all the estimations of medical and clinical human resources were outlined and explained to the medical education system policymakers and were completed using their feedback. Another policy recommendation was designed and formulated on hospital bed distribution and forwarded to the deputy for health care matters at the Ministry of Health. As a result, the Health Roadmap was accepted by Iran's health care system and the medical education system simultaneously without disagreements.

\section{Policy legislation}

The forceful influence of policies on the health system is undeniable. Legal process is an important stage at the end of the policymaking process (14). Agreements were made between the MOHME and the heads of all the medi- 
cal universities to ensure the smooth progress of policy legislation. This roadmap will play a major role in the medical student training system because of student admission capacity decision-making considerations. Furthermore, an understanding was reached and an agreement was made between the Ministry of Health and the Programming and Budgeting Organization to consider the implementation costs of these estimated resources as a part of the national annual budget planning.

\section{Conclusion}

The project report is documented in 11 volumes and is available to all managers at various levels in the Ministry of Health and to all medical universities as well as the relevant national and local authorities outside the Ministry of Health.

\section{Conflict of Interests}

The authors declare that they have no competing interests.

\section{References}

1. Bernard D, Cowan C, Selden T, Cai L, Catlin A, Heffler S. Reconciling medical expenditure estimates from the MEPS and NHEA, 2007. Medicare Medicaid Res Rev. 2012;2(4).

2. Jagger C, Matthews R, Matthews F, Robinson T, Robine JM, Brayne C. The burden of diseases on disability-free life expectancy in later life. J Gerontol Series A: Biol Sci Med Sci. 2007;62(4):408-14.

3. Hajizadeh M, Nghiem HS. Hospital care in Iran: an examination of national health system performance. Intl $\mathrm{J}$ Healthcare Manag. 2013;6(3):201-10.

4. Kumar A, Jiao RJ, Shim S, editors. Predicting bed requirement for a hospital using regression models. Industrial Engineering and Engineering Management, 2008 IEEM 2008 IEEE International Conference on; 2008: IEEE.

5. Abolhallaje M, Mousavi SM, Anjomshoa M, Nasiri AB, Seyedin H, Sadeghifar J, et al. Assessing Health Inequalities in Iran: A Focus on the Distribution of Health Care Facilities. Glob J Health Sci. 2014;6(4):285-91.

6. Bertone MP, Witter S. The complex remuneration of human resources for health in low-income settings: policy implications and a research agenda for designing effective financial incentives. Hum Resour Health. 2015;13:62.

7. Matsumoto M, Inoue K, Bowman R, Kajii E. Self-employment, specialty choice, and geographical distribution of physicians in Japan: A comparison with the United States. Health Policy. 2010;96(3):23944.

8. Dehnavieh R, Mirshekari N, Ghasemi S, Goudarzi R, Haghdoost AA, Mehrolhassani MH, et al. Health technology assessment: Off-site sterilization. Med J Islam Repub Iran. 2016;30(1):299-309.

9. Eftekharizadeh F, Dehnavieh R, Hekmat SN, Mehrolhassani MH. Health technology assessment on super oxidized water for treatment of chronic wounds. Medical J Islam Repub Iran. 2016;30:384.

10. Dehnavieh R, Hekmat SN, Ghasemi S, Mirshekari N. The vulnerable aspects of application of "Health Technology Assessment". Intl J Tech Assess Health Care. 2015;31(03):197-8.

11. Schaik J. A forecasting model for the requests for MRI scans: University of Twente; 2015.p.119-130.

12. Kingdon JW, Thurber JA. Agendas, alternatives, and public policies: Little, Brown Boston; 1984.p.34-89.

13. Sun J, Luo H. Evaluation on equality and efficiency of health resources allocation and health services utilization in China. Intl $\mathrm{J}$ Equit Health. 2017;16(1):127.

14. Teitelbaum JB, Wilensky SE. Essentials of health policy and law: Jones \& Bartlett Publishers; 2016.p. 10-130. 